\section{Comments on Highway Speeds}

\section{Communication}

\author{
R. N. Janeway, Janeway Engineering Company, \\ Detroit, Mich.
}

RECENTLY I received a reprint of the article "Highway Risks at Extreme Speeds," by Dr. Irwin D. J. Bross, which appeared in Public Health Reports, vol. 78, January 1963 . The purpose of this letter is to protest in the strongest possible terms the author's reference to technical material which I have published as authority for his conclusions.

In his article, Dr. Bross attempts to prove that inherent vehicle unmanageability is primarily responsible for prevailing high-speed single car accidents by general reference to my paper, "Vehicle Design Aspects of Safe Handling." This paper was presented at the Conference on Passenger Car Design and Highway Safety, sponsored jointly by the Association for the Aid of Crippled Children and the Consumers Union, and it was published in the conference proceedings. Dr. Bross was also a participant in the conference.

Dr. Bross' entire thesis hinges on a misinterpretation of my data, which he sums up as follows (bottom of p. 29): "The crucial point is this: As the accelerative forces increase, the standard understeer car changes its handling characteristics in the direction of oversteer (3)." The vital point that Dr. Bross disregards, in jumping to this conclusion, is that the typical understeer car never reaches the oversteering condition.

A survey of current automotive practice, in regard to handling stability, is presented in figure 1 (fig. 12 in the conference proceedings). Stability criteria are presented in the form of two curves showing the required relative curb weight distribution between front and rear wheels for varying total absolute car weight, respectively, for neutral steer, and for 5 percent understeer margin. (Neutral steer and understeer or oversteer margin are defined in terms of the location of the resultant of lateral tire thrust forces, with front wheels at the nominal steering angle on a curve. In neutral steer, the resultant acts at the center of gravity ; in understeer, the margin is measured by the distance of resultant aft of c.g. in percent of wheelbase; in oversteer, the margin is distance forward of c.g. in percent of wheelbase.) All U.S. and most foreign front engine standard sedan models are represented by points plotted in relation to these curves.

The calculated criteria assume the following conservative conditions: $(a)$ full passenger and luggage load; $(b)$ uniform tire inflation pressure, as required for maximum wheel loading; (c) average height of center of gravity and roll axis position, for independent front suspension and solid rear axle; $(d)$ side to side weight transfer corresponding to centrifugal acceleration of $0.3 \mathrm{~g} ;(e)$ relatively unfavorable variation in cornering force versus load; $(f)$ zero rear axle steer angle.

Virtually all these designs will be seen to have a positive stability margin, without benefit of rear wheel understeer. In all but four domestic cars, sufficient understeer margin is indicated to maintain at least neutral steer up to $0.5 \mathrm{~g}$ acceleration.

My data bring out the fact that the tire cornering characteristics are such that the weight transfer effect does tend to reduce the understeer margin as centrifugal force increases. However, I also stress the effectiveness of rear axle steer in opposing this tendency, so as to maintain a safe understeer characteristic. Provision for compensating rear axle steer is a standard feature of conventional rear suspension designs, as discussed fully in my paper (pp. 40-42, figs. 9a and 9b). Theoretically, rear wheel understeer can be provided in any amount required to maintain understeer. However, it is undesirable practically to employ more than a fraction of a degree of rear wheel angularity by the conventional method, for reasons discussed on page 42 of the proceedings.

Nevertheless, an inherently stable car, as defined by the criteria shown in figure 1 , is sensitive in its response to small rear wheel steer angles. For example, $0.37^{\circ}$ rear steer angle will provide a 5 percent understeer margin at $0.3 \mathrm{~g}$ acceleration in a car with 45 percent of total weight on front wheels, and $0.27^{\circ}$ will maintain neutral steer for an increase in centrifugal acceleration from $0.3 \mathrm{~g}$ to $0.5 \mathrm{~g}$. These values 
are taken from curves which I included in the paper as originally submitted to the conference participants.

Consequently, with the minimum likely amount of rear wheel steer incorporated in the rear suspensions, none of the domestic cars and few of the foreign cars represented would ever develop oversteer up to the limit of steering force set by maximum adhesion coefficient between tire and road. Hence, my own conclusion, as stated in the Summary: Cornering Stability (p. 26): "Virtually all makes and models of front engine passenger cars, except station wagons, come within the recommended limits of weight distribution for inherent cornering stability."

It should also be noted that oversteering, while undesirable in the hands of the average driver, is by no means synonymous with unmanageability. My paper brings out clearly that oversteering introduces the possibility of complete loss of control if the critical speed is reached at high accelerations in cornering or suddenly changing direction. For example, figure 2 (fig. 13 in proceedings) shows that at $0.5 \mathrm{~g}$ acceleration, even with a 2 percent oversteer margin, critical loss of control would not occur below $90 \mathrm{mph}$ in a 120 -inch wheelbase car.

Figure 1. Required weight distribution vs. total car curb weight for full load stability

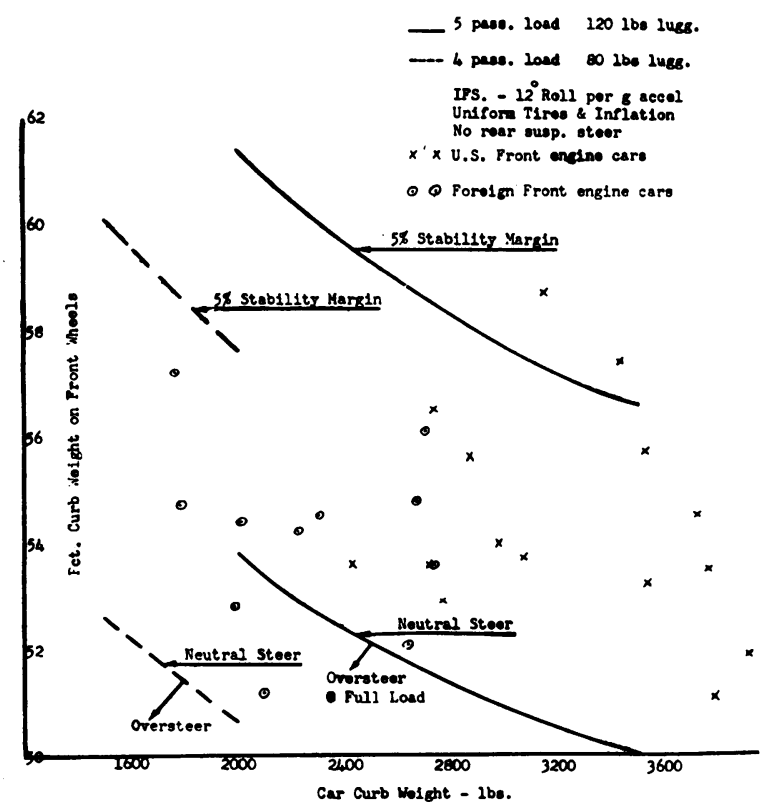

Figure 2. Critical speed vs. oversteer margin

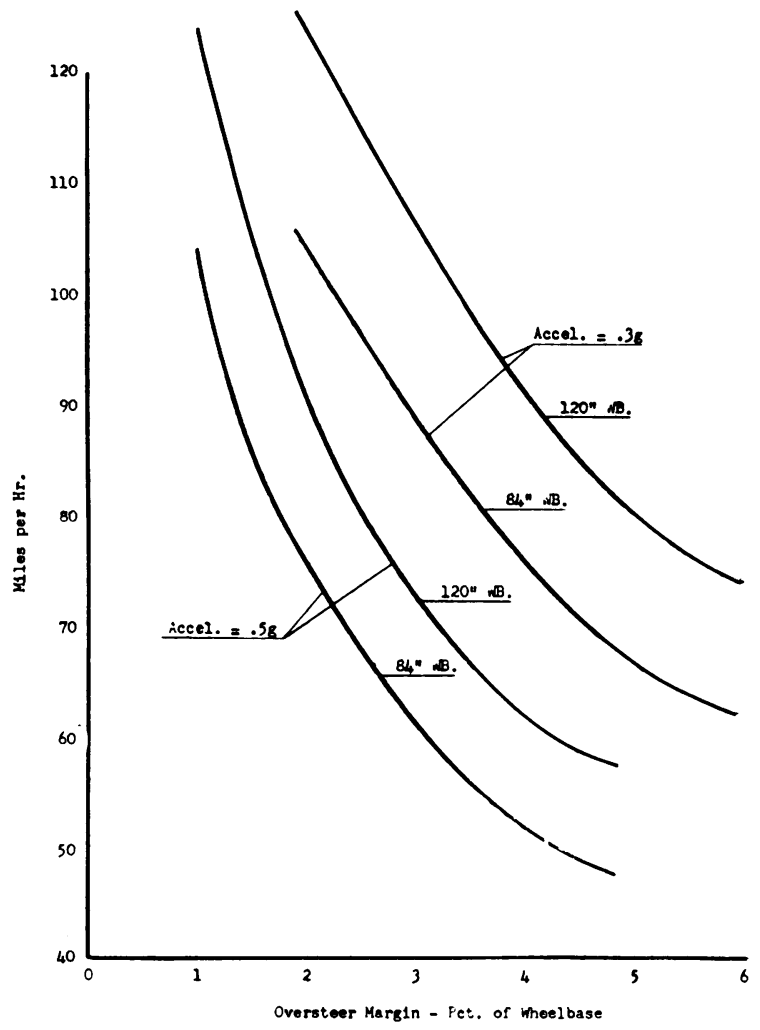

Also, it cannot be emphasized too strongly that maximum maneuverability of a road vehicle is dependent on the tractive coefficient between the wheels and the road. To quote from the introduction of my paper:

Even if the ideal design were to be attained, it could do no more than build into the vehicle an inherent directional stability and ease of control within the limits of adhesion between the tires and the road surface. It is evident not only that the coefficient of adhesion determines the maximum force reaction which can be exerted by the road on the vehicle in controlling its behavior, but also that it is subject to variations in road and weather conditions which are entirely extraneous to the vehicle itself. Here there is no substitute for the driver's judgment and skill in observing the control limitations presented. . . .

For example, at $70 \mathrm{mph}$, with a coefficient of 0.8 , corresponding to good dry road, the smallest possible turning radius would be 500 feet on a flat curve. Regardless of the degree of understeer, under these conditions a driver's attempt to turn about a smaller radius would result in sliding of the front wheels. If the road curvature were sharper than the limiting radius, 
the car would run off the road to the outside of the curve. The centrifugal force would still be less than the overturning value in the average car. Rollover would occur only if the car encountered a negative lateral slope as it left the road.

Dr. Bross' snow analogy (p. 30) comes closer than he realizes to the truth of what happens on dry roads, except that high speed is not a requisite to inducing extreme accelerations beyond the adhesion limit. Centrifugal acceleration is quantitatively equal to $\frac{v^{2}}{r}$ where $v=$ velocity and $r=$ radius of curved path. Therefore, a given acceleration is attainable at low speed if the radius of curvature is small enough. Thus, at $25 \mathrm{mph}$ with 0.8 adhesion coefficient, the control limit will be reached (on level surface) at a 64-foot radius as compared with a 500 -foot radius at $70 \mathrm{mph}$.

There is no doubt that high speed does increase accident hazards. But this is not due to inherent unmanageability of the vehicle, but is a matter of driver limitation combined with the rapid multiplication of energy stored in the moving mass as the speed increases. For the driver, this means that the time margin for error diminishes until it may become smaller than his reaction lag. For the vehicle, the greater amount of energy to be dissipated means a longer distance to decelerate in an emergency and greater potential damage to car and occupants.

Since Dr. Bross' argument is built upon a false premise, his suggestions with regard to legislated manageability standards have no validity. The incompetent driver still remains the principal hazard. In my opinion, any extension of present governmental efforts at accident reduction could best take the form of intensified screening of driver licensees to eliminate the physically or mentally unfit.

\section{REPLY}

Irwin D. J. Bross, Ph.D., director of biostatistics, Roswell Park Memorial Institute, Buffalo, N.Y.

DESPITE appearances to the contrary, Mr. Janeway and $I$ are actually in substantial agreement. The crux of the difficulty is a mat- ter of language. In my original paper I tried to avoid this difficulty by making the following distinction :

The key word here is "manageability." This term is meant to apply to an actual system: the driving population in the United States, the cars that are on the road, and the present highway system. This is a somewhat different concept of the term than is conventional in automotive engineering. For example at high g's a vehicle, in theory, becomes "absolutely unmanageable" when the steering wheel angle has no effect on the course of the vehicle. . . I Inow of no actual demonstration of this phenomenon either on the test track or on the highway. However, long before a vehicle became absolutely unmanageable it would become unmanageable in the hands of an ordinary driver.

Throughout his letter Mr. Janeway refers exclusively to "unmanageability" in this absolute sense and he supposes that I was likewise talking about "inherent vehicle unmanageability." I wasn't. Indeed, I had repeatedly stressed that it was the system concept, rather than the vehicle concept, that is important in the public health problem of highway accidents.

The one sentence from my paper which Mr. Janeway specifically quotes is:

"The crucial point is this: As the accelerative forces increase, the standard understeer car changes its handling characteristics in the direction of oversteer (3)."

I have underlined the portion of the sentence that enters into the subsequent argument for systems-unmanageability. It was a mistake on my part to use the engineering jargon in the remainder of this sentence. For engineering readers this jargon would convey the erroneous impression that "unmanageability" is part of the same jargon. Hence they would suppose that the attainment of inherent-vehicle unmanageability is necessary for my argument, whereas it is the change in handling characteristics that is important in systems-unmanageability.

In my original paper I used a snow-driving analogy to give a more vivid picture of system. I pointed out that "As long as a car is driven at a fairly constant speed without sharp turns, the car performs in snow much as it would on a dry highway. However, in sharp turns or abrupt braking where stronger accelerative forces come into play, the car becomes unman- 